\title{
Nonperturbative expression for the transmission through a leaky chiral edge mode
}

\author{
Kun Woo Kim, ${ }^{1}$ Israel Klich, ${ }^{2}$ and Gil Refael ${ }^{1}$ \\ ${ }^{1}$ Department of Physics, California Institute of Technology, 1200 East California Boulevard, MC 114-36, Pasadena, California 91125, USA \\ ${ }^{2}$ Department of Physics, University of Virginia, Charlottesville, Virginia 22904, USA
}

(Received 27 July 2013; revised manuscript received 12 February 2014; published 24 March 2014)

\begin{abstract}
Chiral edge modes of topological insulators and Hall states exhibit nontrivial behavior of conductance in the presence of impurities or additional channels. We present a simple formula for the conductance through a chiral edge mode coupled to a disordered bulk. For a given coupling matrix between the chiral mode and bulk modes, and a Green's function matrix of bulk modes in real space, the renormalized Green's function of the chiral mode is expressed in closed form as a ratio of determinants. We demonstrate the usage of the formula in two systems: (i) a 1d wire with random on-site impurity potentials for which we found that the disorder averaging is made simpler with the formula, and (ii) a quantum Hall fluid with impurities in the bulk for which the phase picked up by the chiral mode due to the scattering with the impurities can be conveniently estimated.
\end{abstract}

DOI: 10.1103/PhysRevB.89.104204

PACS number(s): 71.23.An, 73.43.Jn, 03.65.Vf

\section{INTRODUCTION}

Interest in the behavior of chiral modes on the boundary of $2 \mathrm{~d}$ insulating systems has been growing in recent years. In particular, such modes are always present on the boundaries of topological states such as topological insulators [1-7] and quantum Hall samples [8-11]. For example, an important consequence is that zero-temperature electron transport along an edge of a topological insulator will have a quantized conductance, if time-reversal symmetry is not broken. To describe realistic systems, however, it is always necessary to take into account imperfections, such as potential disorder or impurity scattering [12-14], or even the presence of bulk states. Interestingly, chiral edge states might even be the result of adding disorder to trivial spin-orbit coupled semiconductors, and the appearance of the so-called Anderson topological insulator [15-17]. In the course of such a disorder-induced transition, the system necessarily carries extended bulk states as well as chiral modes. Such topological metal systems show a nontrivial behavior of transport properties $[18,19]$.

Disorder effects in nonchiral 1d systems, e.g., localization phenomena due to the introduction of random potential, were also studied theoretically and computationally for many years (for a review see, e.g., [20]). While, computationally, it is straightforward to confirm localization of wave functions in 1d single-particle systems, the theoretical studies of localization properties and transitions in $1 \mathrm{~d}$ and higher have proven challenging. A scaling theory suggested in [21] shows the localization of $1 \mathrm{~d}$ and $2 \mathrm{~d}$ at any weak disorder in the system. For a given distribution of random disorder, an upper bound on localization length was found in [22]. Powerful disorderaveraging techniques are available, such as the supersymmetry approach [23] and the replica trick [24], which induces an effective nonlinear sigma field theory. Also, in strongly disordered systems such as random spin chains, trap models, and random polymers, the role of quenched disorder on quantum and thermal fluctuations has been studied by the strong-disorder renormalization group method (for a review see, e.g., [25]).

Considerable advances, however, can be made by simple and, arguably, more reliable algebraic methods, when considering a $1 \mathrm{~d}$ chiral mode connected to a bulk. Such an approach could apply to intrinsic chiral edge modes, and even to localization in nonchiral 1d systems. An example of such a simplification is the subject of the present paper.

Here we present a closed-form Green's function of a chiral mode coupled to a bulk and its applications. In Sec. II, the Green's function is expressed as a ratio of determinants, and involving in the Green's function of the bulk and arbitrary couplings between the chiral mode and bulk. An application to a disordered strict $1 \mathrm{~d}$ wire is introduced in Sec. III. The 1d wire is modeled in terms of two chiral modes, and the "det formula" is applied in the presence of on-site impurities. The disorder averaging of the transmission coefficient, the logarithm of the transmission coefficient, and the reflection coefficient are worked out in the subsections. In Sec. IV, the phase picked up by the chiral mode in a quantum Hall fluid system is studied. We conclude in Sec. V. Additional details of disorder averaging of the logarithm of the transmission coefficient are worked out in the Appendix.

\section{GREEN'S FUNCTION OF A LEAKY CHIRAL MODE}

In this section, we derive an analytic expression for the Green's function between the two ends of a chiral mode coupled to bulk modes (see Fig. 1). Throughout the paper, we assume that the scattering happens at discrete locations, allowing us to turn the problem into a matrix problem. We therefore only need the Green's function of the right-moving chiral mode at the scattering positions:

$$
\left(G_{R}\right)_{n m}=\frac{1}{i v_{F}} e^{i k|n-m| a} \theta(n-m),
$$

which in a real-space basis is a left lower triangular matrix. We define $\theta(0)=1 / 2$, and $n, m=1, \ldots, N$ are indices of scattering sites. We assume that the scattering centers are equally spaced. This assumption can be lifted by modifying the translational operator $\hat{U}_{t}$ introduced later. The chiral Green's function renormalized by the coupling with the bulk modes represented by $G_{B}$ can be obtained by writing the Hamiltonian of the system in block form and using standard block inversion, 


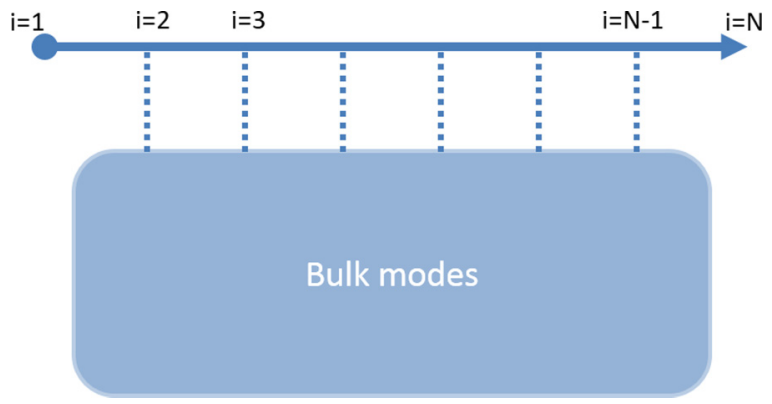

FIG. 1. (Color online) A chiral mode is coupled to a bulk that may contain localized and propagating modes. The chiral Green's function from site $i=1$ to $i=N$ is found for a given coupling between the chiral and bulk modes expressed in matrix $T$ in real space, and bulk Green's function $G_{B}$ in Sec. II.

with the result

$$
\mathcal{G}=\frac{1}{G_{R}^{-1}-V G_{B} V^{\dagger}}
$$

where $V$ represents couplings between the chiral mode and bulk modes. For convenience, we pull out phase factors from the Green's function, writing it as

$$
G_{R}=\hat{U}_{t}^{\dagger} G_{R 0} \hat{U}_{t} .
$$

Here $\hat{U}_{t}=e^{-i k a \hat{n}}$, where $\hat{n}$ is the position operator along the wire, $\hat{n}_{n m}=\delta_{m n} n$, and $G_{R 0}$ is a Green's function with zero wave number in Eq. (1), which can be thought of as the chiral Green's function with the phase factors gauged out and without coupling to bulk modes. Due to the particular form of $G_{R 0}$, we can write its inverse explicitly, finding that

$$
G_{R 0}^{-1}=-4 v_{F}^{2} \hat{U}_{s} G_{R 0} \hat{U}_{s}^{\dagger},
$$

where a sign operator $\hat{U}_{s}=e^{i \pi \hat{n}}$ was introduced. We note that $G_{R}$, as well as its inverse $G_{R}^{-1}$, are lower triangular matrices. With these definitions, we now have

$$
\mathcal{G}=\frac{1}{4 v_{F}^{2}} \hat{U}_{s t} \frac{1}{-G_{R 0}-\mathcal{T} G_{B} \mathcal{T}^{\dagger}} \hat{U}_{s t}^{\dagger},
$$

where $\hat{U}_{s t}=\hat{U}_{s} \hat{U}_{t}$, and $\mathcal{T}=\hat{U}_{s t} V / 2 v_{F}$.

Our main interest in this paper is to get the Green's function between two ends, described by $\mathcal{G}_{N 1}$. To do so, we use Cramer's rule, i.e., $C_{i j}^{-1}=(-1)^{i+j} M_{j i} \operatorname{det}(C)^{-1}$, where $M_{j i}$ is the $j i$ th minor of the matrix $C$, together with Eq. (5), and find

$$
\begin{aligned}
\mathcal{G}_{N 1} & =(-1)^{N} \frac{e^{i(N-1) k a}}{4 v_{F}^{2}}\left(\frac{1}{G_{R 0}+\mathcal{T} G_{B} \mathcal{T}^{\dagger}}\right)_{N 1} \\
& =(-1)^{N} \frac{e^{i(N-1) k a}}{4 v_{F}^{2}} \frac{(-1)^{N+1} M_{1 N}}{\operatorname{det}\left[G_{R 0}+\mathcal{T} G_{B} \mathcal{T}^{\dagger}\right]},
\end{aligned}
$$

where the $(1, N)$ th minor of the matrix is

$$
\begin{aligned}
M_{1 N} & =\operatorname{minor}_{1 N}\left[G_{R 0}+\mathcal{T} G_{B} \mathcal{T}^{\dagger}\right] \\
& =4 i v_{F}(-1)^{N} \operatorname{det}\left[-G_{R 0}^{T}+\mathcal{T} G_{B} \mathcal{T}^{\dagger}\right] \\
& =4 i v_{F} \operatorname{det}\left[G_{R 0}^{T}-\mathcal{T} G_{B} \mathcal{T}^{\dagger}\right] .
\end{aligned}
$$

Here, we manage to express the minor in the symmetric form of the denominator in Eq. (7). The derivation is detailed in Fig. 2.
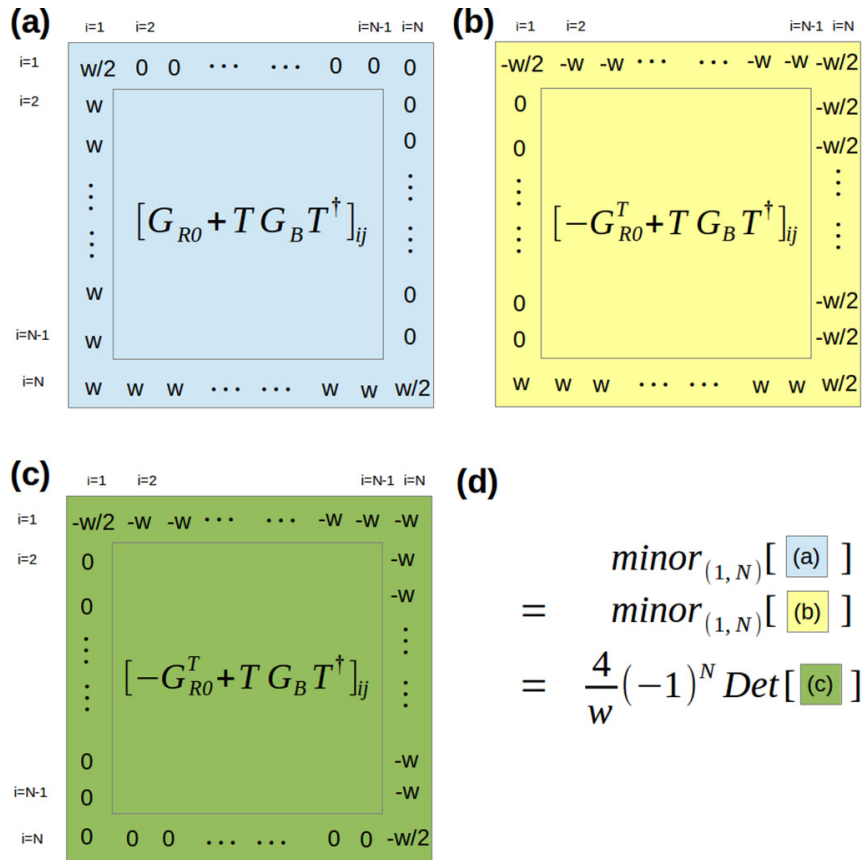

(d)

$$
\begin{aligned}
& \operatorname{minor}_{(1, N)}[\text { (a) }] \\
= & \operatorname{minor}_{(1, N)}[\text { (b) }] \\
= & \frac{4}{w}(-1)^{N} \operatorname{Det}[\text { (c) }]
\end{aligned}
$$

FIG. 2. (Color online) The derivation of Eq. (10) is detailed with diagrams using $w=1 / i v_{F}$, for simplicity. Note that $\mathcal{T} G_{B} \mathcal{T}^{\dagger}$ has no element in the outer part of the matrix since there is no coupling at site $i=1$ and $i=N$. The $(1, N)$ th minor of matrix (a) is the same with that of matrix (b), which is obtained by subtracting the last row of (a) from all other rows. Then, consider matrix (c) which has the outer elements of $-G_{R 0}^{T}$. Its determinant is the same with the $(1, N)$ th minor of matrix (b) up to a constant as expressed in (d).

As a result, the renormalized Green's function is expressed in terms of the ratio of two determinants:

$$
\mathcal{G}_{N 1}=\mathcal{G}_{N 1}^{(0)} \frac{\operatorname{det}\left[G_{L 0}-\mathcal{T} G_{B} \mathcal{T}^{\dagger}\right]}{\operatorname{det}\left[G_{R 0}+\mathcal{T} G_{B} \mathcal{T}^{\dagger}\right]},
$$

where $\mathcal{G}_{N 1}^{(0)}=e^{i(N-1) k a} / i v_{F}$, and $G_{R 0}^{T}$ is replaced by $G_{L 0}$, which is the Green's function of a left-moving chiral mode: $\left(G_{L 0}\right)_{m n}=\left(G_{R 0}\right)_{n m}$. Note that $\mathcal{T}$ is essentially the coupling matrix between the chiral and bulk modes, but rescaled by the Fermi velocity and multiplied by the sign and translational matrices. We thus obtained the renormalized chiral Green's function coupled with bulk modes in a compact and closed form. The additional factor is expressed as a ratio of two determinants, and we find it particularly advantageous to perform disorder averaging. We continue the detailed calculation of transmission of disordered $1 \mathrm{~d}$ wire in the following section.

\section{EXAMPLE: DISORDERED 1D WIRE}

Our first example is a $1 \mathrm{~d}$ wire with random on-site potentials first considered by Anderson [26]. The physical quantity of interest is the transmission coefficient of the system, as it is the most relevant for $1 \mathrm{~d}$ and quasi-1d wires connected to leads [27], rather than the conductance of the system, which is the ratio of the transmission and reflection coefficients. Our expression for the Green's function is not directly applicable to this problem, since the $1 \mathrm{~d}$ wire does not contain a chiral mode explicitly. Nevertheless, we show in Sec. III A that the 
(a)

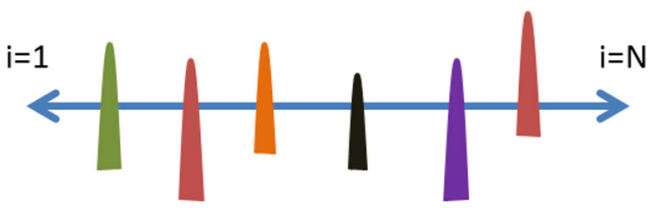

(b)

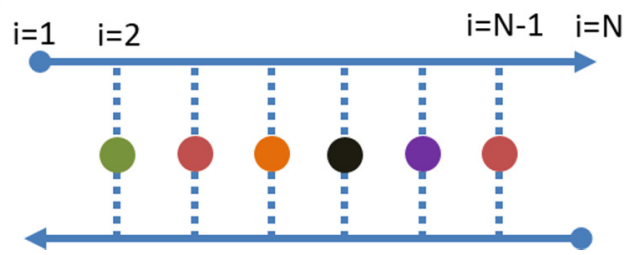

FIG. 3. (Color online) (a) 1d wire with random on-site impurities. (b) An equivalent model of (a). The 1d clean bulk mode is described by two chiral modes, and random impurities are modeled by the coupling between two chiral modes through quantum dots with random on-site potentials; see Eq. (17).

disordered $1 \mathrm{~d}$ wire can be equivalently modeled by two chiral modes with the couplings through quantum dots. We then apply the det formula in Sec. III B to find the transmission coefficient for a given realization of random impurities. In Sec. III C, the disorder averaging of the transmission coefficient $T$, as well as of $\log (T)$, and $1 / T$ are computed. We also show that our approach to computing the transport quantities provides a clear picture for why the logarithm of the transmission is a well behaved statistical quantity. Plus, it simplifies the process of disorder averaging.

\section{A. Alternative model of disordered 1d wire}

In this section we consider an ordinary $1 \mathrm{~d}$ wire, which, using the results of the previous section, we decompose into two chiral modes coupled through a series of quantum dots. The system contains $(N-2)$ impurities with random strengths which is expressed by matrix $\alpha$ in real space (see Fig. 3):

$$
(\alpha)_{n m}=\delta_{n m} \alpha_{n},
$$

where index $n$ is for the scattering sites. The first site, $n=1$, and the last site, $n=N$, play the role of leads connected to the disordered wire from both ends.

The Green's function through the disordered 1d wire can be cast in a $T$-matrix formulation as

$$
\mathcal{G}_{N 1}=\left(G+G \frac{1}{I-\alpha G} \alpha G\right)_{N 1},
$$

where $G$ is the free Green's function on the wire. We consider a system with $N$ scattering points and placed at intervals of length $a$. Only positions on the wire at which scattering occurs contribute in a nontrivial way in Eq. (13); therefore we can replace the continuous $G$ with an $N \times N$ matrix with elements

$$
(G)_{n m}=\frac{1}{i v_{F}} e^{i k|n-m| a} .
$$

Now consider chiral Green's functions with left and right moving modes. One can relate them to the nonchiral Green's function by

$$
G=G_{R}+G_{L},
$$

where the left-moving chiral Green's function is similar to Eq. 1 with $m$ and $n$ exchanged in the step function; therefore, $\left(G_{L}\right)_{m n}=\left(G_{R}\right)_{n m}$. Next, we express the Green's function through the disordered 1d system, Eq. (13), in terms of the chiral modes:

$$
\mathcal{G}_{N 1}=\left(G_{R}+G_{R} \frac{1}{I-\alpha\left(G_{R}+G_{L}\right)} \alpha G_{R}\right)_{N 1} .
$$

Note that except for the Green's function in the $T$ matrix, the Green's function $G$ on the left and on the right have been replaced by $G_{R}$, which is justified since $(G)_{n m}=\left(G_{R}\right)_{n m}$ for $n>m$, while $\alpha_{n}$ is nonzero only at the points $N-1>n>1$.

Finally, we obtain a model of a disordered 1d wire by introducing quantum dots replacing random on-site potentials, and the two chiral modes are connected to the dots through a coupling matrix $V$ :

$$
\alpha=V G_{Q} V^{\dagger}
$$

where $G_{Q}$ is the Green's function of quantum dots possessing random chemical potentials. By substituting the random potential matrix $\alpha$ in Eq. (16), now $\mathcal{G}_{N 1}$ describes the Green's function of right-moving chiral mode as in Fig. 3(b).

Note that all of our following analysis is performed in the parameter range where the dispersion relation can be treated as linear. More specifically, consider a dispersion relation at some momentum:

$$
\begin{aligned}
E(k)= & E\left(k_{0}\right)+v_{F}\left(k-k_{0}\right)+u_{2}\left(k-k_{0}\right)^{2} \\
& +u_{3}\left(k-k_{0}\right)^{3}+O\left(k^{4}\right) .
\end{aligned}
$$

To justify the validity of the det formula, which is based on the linear dispersion of a chiral mode, we must have $k-k_{0} \ll$ $\Delta k_{c}=\min \left(v_{F} / u_{2}, \sqrt{v_{F} / u_{3}}\right)$. Therefore, the impurity strength should be less than $v_{F} \Delta k_{c}$. On the other hand, we introduce the limit of weak and strong impurity strength by the expansion parameters and the number of impurities:

$$
\begin{aligned}
\alpha & \ll \frac{v_{F}}{N} \quad \text { (weak disorder), } \\
\frac{v_{F}}{N} & \ll \alpha \ll v_{F} \Delta k_{c} \quad \text { (strong disorder). }
\end{aligned}
$$

In the following sections, we refer to the weak and strong limits of impurity strength according to the above determination. For some systems with $v_{F} / N \geqslant v_{F} \Delta k_{c}$, we disregard the strongdisorder regime.

\section{B. Green's function through disordered 1d wire}

Consider a $1 \mathrm{~d}$ wire with a particular disorder realization, Fig. 3(b). We would like to compute the transmission through the wire from site $i=1$ to $i=N$; therefore the bulk corresponding to the one in Fig. 1 is the quantum dots coupled with a left-moving chiral mode. The Green's function of the bulk is

$$
G_{B}=\frac{1}{G_{Q}^{-1}+V^{\dagger} G_{L} V},
$$

where the left-moving chiral Green's function renormalizes the Green's function of quantum dots. Note the hopping matrix $V$ 
has now a different order compared to Eq. (2). For our purpose, it is convenient to consider with a simplified form:

$$
G_{L}=-\frac{1}{4 v_{F}^{2}} \hat{U}_{s t}^{\dagger} G_{L 0}^{-1} \hat{U}_{s t} .
$$

Define $\mathcal{T}_{2}=\hat{U}_{s t}^{\dagger} V / 2 v_{F}$, and plug in the left-moving chiral Green's function back to the bulk Green's function expression:

$$
G_{B}=\frac{1}{G_{Q}^{-1}+\mathcal{T}_{2}^{\dagger} G_{L 0}^{-1} \mathcal{T}_{2}} .
$$

Next, let us work with the renormalized Green's function for this bulk system. Equation (11) is reduced to

$$
\begin{aligned}
\frac{\mathcal{G}_{N 1}}{\mathcal{G}_{N 1}^{(0)}} & =\frac{\operatorname{det}\left[G_{L 0}-\mathcal{T} G_{B} \mathcal{T}^{\dagger}\right]}{\operatorname{det}\left[G_{R 0}+\mathcal{T} G_{B} \mathcal{T}^{\dagger}\right]} \\
& =\frac{\operatorname{det}\left[G_{L 0}\left(\mathcal{T} G_{B} \mathcal{T}^{\dagger}\right)^{-1}-I\right]}{\operatorname{det}\left[G_{R 0}\left(\mathcal{T} G_{B} \mathcal{T}^{\dagger}\right)^{-1}+I\right]} \\
& =\frac{\operatorname{det}\left[G_{L 0}-\left(I-G_{L 0} \hat{U}_{t}^{2} G_{L 0}^{-1} \hat{U}_{t}^{\dagger 2}\right) \mathcal{T} G_{Q} \mathcal{T}^{\dagger}\right]}{\operatorname{det}\left[G_{R 0}+\left(I+G_{R 0} \hat{U}_{t}^{2} G_{L 0}^{-1} \hat{U}_{t}^{\dagger 2}\right) \mathcal{T} G_{Q} \mathcal{T}^{\dagger}\right]} \\
& =\frac{\operatorname{det}\left[G_{L 0}^{\prime}-R \mathcal{T} G_{Q} \mathcal{T}^{\dagger}\right]}{\operatorname{det}\left[G_{R 0}^{\prime}+\mathcal{T} G_{Q} \mathcal{T}^{\dagger}\right]},
\end{aligned}
$$

where in the second equality the determinant with lower case is designated as the determinant of matrix excluding the boundary elements. Next, the inverse of $\mathcal{T} G_{B} \mathcal{T}^{\dagger}$ is expressed using the relation $\mathcal{T}_{2} \mathcal{T}^{-1}=\hat{U}_{t}^{\dagger 2}=e^{i 2 k a \hat{n}}$ and $\mathcal{T} G_{Q} \mathcal{T}^{\dagger}$ recovering a determinant of a whole matrix. In the last equality, new Green's functions have been defined:

$$
\begin{aligned}
G_{R 0}^{\prime} & =\frac{1}{G_{R 0}^{-1}+\hat{U}_{t}^{2} G_{L 0}^{-1} \hat{U}_{t}^{\dagger 2}} \\
& =-\frac{1}{4 v_{F}^{2}} \hat{U}_{s t} \frac{1}{G_{R}+G_{L}} \hat{U}_{s t}^{\dagger},
\end{aligned}
$$

where $G_{R}+G_{L}$ is the Green's function of a clean 1d wire Hamiltonian with nearest-neighbor hopping; therefore the inverse is a tridiagonal matrix. On the other hand,

$$
\begin{aligned}
G_{L 0}^{\prime} & =\frac{1}{I+G_{R 0} \hat{U}_{t}^{2} G_{L 0}^{-1} \hat{U}_{t}^{\dagger 2}} G_{L 0} \\
& =G_{R 0}^{\prime} G_{R 0}^{-1} G_{L 0}
\end{aligned}
$$

and

$$
\begin{aligned}
R & =\frac{1}{I+G_{R 0} \hat{U}_{t}^{2} G_{L 0}^{-1} \hat{U}_{t}^{\dagger 2}}\left(I+G_{L 0} \hat{U}_{t}^{2} G_{L 0}^{-1} \hat{U}_{t}^{\dagger 2}\right) \\
& =I-G_{R 0}^{\prime}\left(I+G_{R 0}^{-1} G_{L 0}\right) \hat{U}_{t}^{2} G_{L 0}^{-1} \hat{U}_{t}^{\dagger 2} .
\end{aligned}
$$

Studying the second term on the right side of Eq. (33), equal to $(I-R)$, we find that $(I-R)_{n m}=0$ for $n>1$, and $(I-$ $R)_{11}=1$. As a result, we find that $(R)_{11}=0$ and $(R)_{n n}=1$ for $n \neq 1$. Finally, one can verify that

$$
\left(G_{L 0}^{\prime}+R G_{R 0}^{\prime}\right)_{n m}=\frac{1}{4 i} \delta_{n, 1} \delta_{m, N},
$$

and the renormalized Green's function is reduced to

$$
\begin{aligned}
\frac{\mathcal{G}_{N 1}}{\mathcal{G}_{N 1}^{(0)}} & =\operatorname{det}\left[\left(G_{L 0}^{\prime}+R G_{R 0}^{\prime}\right) /\left(G_{R 0}^{\prime}+\mathcal{T} G_{Q} \mathcal{T}^{\dagger}\right)-R\right] \\
& =\frac{1}{\operatorname{det}\left[I+\mathcal{T} G_{Q} \mathcal{T}^{\dagger} G_{R 0}^{\prime-1}\right]} \\
& =\frac{1}{\operatorname{det}\left[I-\alpha\left(G_{R}+G_{L}\right)\right]},
\end{aligned}
$$

using the notation previously defined in the alternative model. A Green's function of a similar form was discovered by Thouless [28] and used to obtain the density of state. Once the transmission of a $1 \mathrm{~d}$ disordered system for particular disorder realization is written in this way, the analytic manipulation becomes easier and the disorder averaging is rendered accessible as we present in the next sections.

\section{Disorder averaging}

The ability to analytically perform disorder averaging for observable quantities can provide vital insights to the behavior of disordered systems. Although a purely 1d system is not experimentally accessible (with the exception, perhaps, of cold atoms [29]), there have been numerous theoretical studies of disordered 1d system in relation to the Anderson localization. Though not present in purely 1d system, the universal fluctuation of conductance can be computed from the disorder averaged moments of conductance [30,31]. And, the localization length is deduced from the disorder averaging of the logarithm of conductance. Lastly, the scaling behavior of disorder averaged resistance with system size has been heavily studied [32-36]. In the following subsections, using the det formula we perform the disorder averaging of transmission, its logarithm, and resistance.

\section{Transmission coefficient: $\bar{T}$}

In this section we find an analytic expression for the transmission coefficient of a disordered wire in the weak disorder limit, and then perform disorder averaging. The explicit expression of the transmission obtained here will be used in later sections. Note that the conductance measured in experiments involves also the connected leads; therefore, the transmission considered here is directly relevant to conductance even for a strict 1d system [27]. One can express the transmission for a particular disorder realization using transfer matrix methods. The resistance is related to a product of transfer matrices, and is thus relatively easy to disorder average, while the transmission is its inverse, which is harder to average. Group-theoretical approaches $[37,38]$ were suggested to perform the disorder average of the transmission, with some success in the weak-disorder limit. We present here an approach that enables obtaining disorder averaging in a simpler fashion, and can possibly be generalized to spatially correlated disorder distribution.

In the weak-disorder limit we make use of the Green's function Eq. 35 to obtain the transmission coefficient through the disordered wire, $T=\left|\mathcal{G}_{N 1}\right|^{2}$. The determinant can be perturbatively expanded using $\operatorname{det}(I+O)=\exp \left[\operatorname{Tr}\left(O-O^{2} / 2+\right.\right.$ $\cdots)$ ]. In our case, the matrix $O$ is simply $-\alpha\left(G_{R}+G_{L}\right)$. We 
now consider the traces:

$$
\begin{aligned}
(O)_{n n} & =-\frac{\alpha_{n}}{i v_{F}} \\
\left(O^{2}\right)_{n n} & =-\sum_{m} \frac{\alpha_{n} \alpha_{m}}{v_{F}^{2}} e^{2 i k a|n-m|} .
\end{aligned}
$$

We see that the diagonal elements of $O$ are purely imaginary; therefore the Tr $O$ term just provides a phase to the Green's function and the transmission coefficient is unchanged by the first-order term. In contrast, the diagonal elements of $O^{2}$ possess a nonvanishing real part, and therefore the leading order contribution in the weak-impurity limit.

For a given realization of impurity strengths $\{\alpha\}$, we have the transmission coefficient:

$$
\begin{aligned}
T(\{\alpha\}) & =\exp \left(-\operatorname{Tr}\left[\sum_{m=1}^{M_{u p}} \frac{(-1)^{m+1}}{m}\left(O^{m}+O^{* m}\right)\right]\right) \\
& \simeq \frac{1}{\exp \left[-\operatorname{Tr}\left(O^{2}+O^{* 2}\right) / 2\right]} \\
& =\frac{1}{\exp \left[\sum_{n, m} \alpha_{n} \alpha_{m} \cos (2 k a|n-m|) / v_{F}^{2}\right]},
\end{aligned}
$$

where the upper bound of sum in the exponent of Eq. (38), $M_{u p}=2$, is taken in the second equality. The indices $n$ and $m$ run over the lattice positions between $n=2$ and $n=N-1$. The bilinear summation over impurity strengths is particularly useful for analytic disorder averaging if the $\alpha_{i}$ are given by a Gaussian distribution:

$$
\begin{aligned}
\bar{T} & =\int T(\{\alpha\}) \prod_{i=2}^{N-1} \frac{e^{-\alpha_{i}^{2} / 2 \sigma^{2}}}{\sqrt{2 \pi} \sigma} d \alpha_{i} \\
& =\frac{1}{\sqrt{\operatorname{det}(K)}},
\end{aligned}
$$

where the matrix $K$ is

$$
(K)_{n m}=\delta_{n m}+2 \sigma^{2} \cos (2 k a|n-m|) / v_{F}^{2} .
$$

$K$ can also be written in the form

$$
K=I_{N-2}+\frac{2 \sigma^{2}}{v_{F}^{2}}\left(|v\rangle\left\langle v|+| v^{*}\right\rangle\left\langle v^{*}\right|\right),
$$

where $|v\rangle$ is a vector with elements $(v)_{n}=e^{i n k a}$ with the index $n=1, \ldots, N-2$. This form shows that $K$ is a rank 2 matrix, and the determinant of $K$ is as easy as taking a determinant of a $2 \times 2$ matrix. As a result:

$$
\operatorname{det}(K)=\left[1-(N-2) \frac{\sigma^{2}}{v_{F}^{2}}\right]^{2}-\frac{\sigma^{4}}{v_{F}^{4}}\left|\Sigma_{n=1}^{N-2} e^{i n k a}\right|^{2} .
$$

In the weak-disorder regime, the first term gives the dominant contribution to the transmission coefficient. Figure 4 shows a surprisingly good agreement between numerics that take into account all orders and the analytics within second-order for different system size $N$. The perturbation theory works only when the expansion parameter is smaller than unity. For our case, from the expansion parameter, $\operatorname{Tr}\left(O^{2}\right)$ in Eq. (37), the condition is

$$
\lambda=\frac{\sigma^{2} N}{v_{F}^{2}}<1
$$

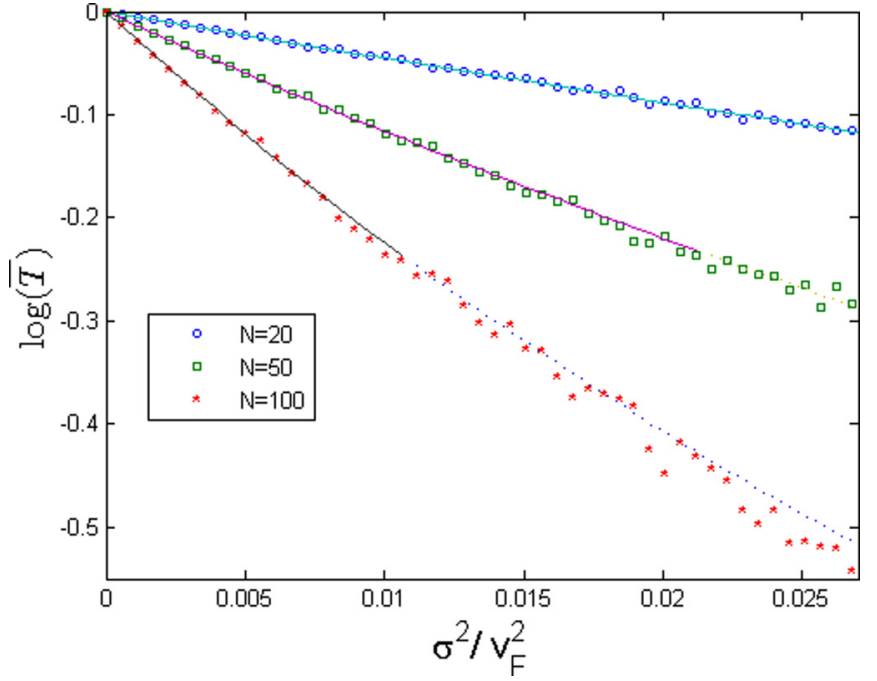

FIG. 4. (Color online) $\log \bar{T}$ of a disordered $1 \mathrm{~d}$ wire with $k a=$ $0.45 \pi$ is plotted. 1000 different disorder realizations are numerically performed (dots) and compared with the analytic expression within the perturbative regime (solid lines) and nonperturbative regime (dashed lines) for different system sizes $N=20,50,100$. The perturbative regime is determined by $\lambda=\sigma^{2} / v_{F}^{2}<1 / N$ according to Eq. (46).

The horizontal axis of the plot is $\sigma^{2} / v_{F}^{2}$, and therefore the analytic expression is valid for $\sigma^{2} / v_{F}^{2}<1 / N$. For the largest system size $N=100$ in the plot, we can see the deviation as early as $\sigma^{2} / v_{F}^{2} \simeq 0.01$, consistent with the discussion.

A natural question is, How will the estimates improve if higher order terms in the matrix $O$ are taken into account? We found that the improvement is negligibly small as shown in Fig. 5, which shows the comparison for the inclusion of different orders. This is an interesting point to discuss. The

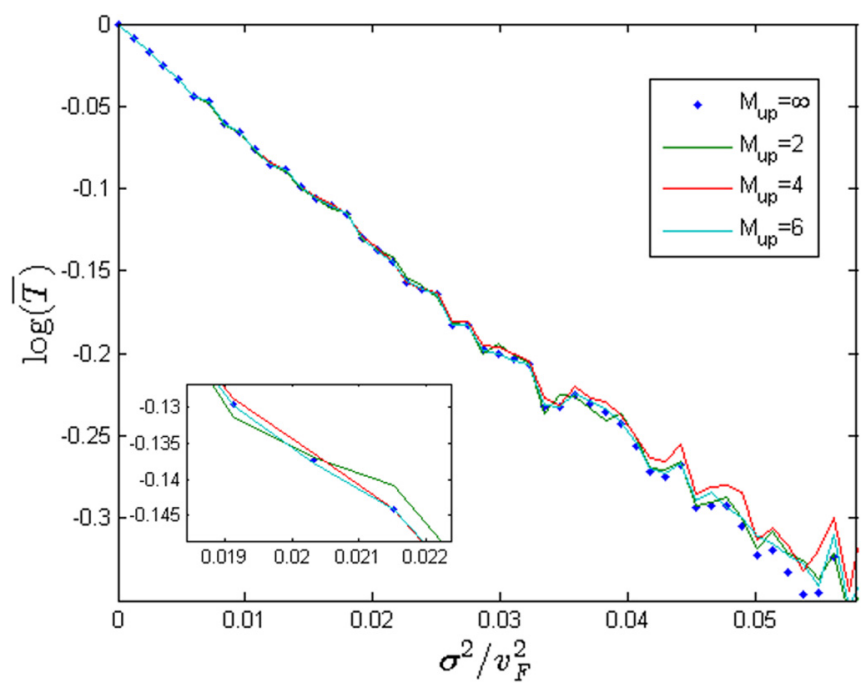

FIG. 5. (Color online) $\log \bar{T}$ with $k a=0.45 \pi$ is numerically computed (lines) considering different upper bounds $M_{u p}$ of summation in the exponent of Eq. (38), and compared with exact numerical results (dots) for a system of $N=30$. The improvement by including the higher order correction is slight. 
random variables, i.e., the strengths of the impurities, $\alpha_{n}$, appear in the exponent of exponential function. Therefore, the average transmission coefficient is dominated by the realizations with very small impurity strengths; the contributions of other realizations are exponentially suppressed. Because at small impurity strengths the contribution of higher order terms in Eq. (38) is also small, we find that the leading order actually gives a good estimate of all orders. Note that for systems with a spatially correlated disorder distribution, we can simply introduce a spatially correlated Gaussian distribution in Eq. (41), which allows the averaging to follow along the same lines as for systems with noncorrelated distributions.

\section{Localization length: $\overline{\log (T)}$}

As shown in the previous section, the disorder average of the transmission is dominated by a few rare realizations with a set of very weak impurities. On the other hand, it is found that the logarithm of the transmission is statistically well behaved [33,39-42]. In the weak-disorder limit, the explicit expression of the inverse localization length was found using a perturbation approach to the first order in disorder strength $[37,42,43]$. In this section, we present the expression of $\overline{\log T}$ which includes all terms linearly proportional to system size for $W / v_{F}<1$. We also obtain the terms which are not linearly proportional to system size in the Appendix. They may have a nontrivial effect on the inverse localization when the electron's wavelength is commensurate with the lattice spacing [42]. We show, using our formalism, why $(\log T) / L$ obeys the central limit theorem. Also, we discuss the behavior of $\overline{\log T}$ in the strong-disorder limit.

Let us first consider the disorder strength within the perturbation regime, $W / v_{F}<1$, where the $W$ is the cutoff of uniform disorder distribution. Here we employ the uniform distribution instead of a Gaussian distribution, ensuring all moments are bounded. Working in the perturbation regime validates the expansion of $\log (I+O)$ for the terms linearly proportional to system size considered below. We consider the terms with even order of $O$ in the expansion, since they are the only nonvanishing terms after disorder averaging. The term with even $m$ th order of $O$ is

$$
\begin{aligned}
& \frac{1}{m} \operatorname{Tr}\left[O^{m}+O^{* m}\right] \\
& \quad=\frac{2}{m} \sum_{n_{1}, \ldots, n_{m}}\left(\prod_{i=1}^{m} \frac{\alpha_{n_{i}}}{v_{F}}\right) \cos \left(k a \sum_{i=1}^{m}\left|n_{i+1}-n_{i}\right|\right),
\end{aligned}
$$

where $n_{i}=2, \ldots, N-1$ is index of impurity site, and $n_{m+1} \equiv$ $n_{1}$. Though the exact summation over indices seems tricky, we find the dominant contribution by summing terms with zero argument of the cosine function: $n_{i+1}=n_{i}$ for all $i$. Retaining such terms, we find that the average of the logarithm transmission is given by

$$
\begin{aligned}
\overline{\log (T)} & \simeq-\frac{L}{a} \sum_{m=1}^{\infty} \int_{-W}^{W}(-1)^{m+1} \frac{1}{m}\left(\frac{\alpha}{v_{F}}\right)^{2 m} \frac{d \alpha}{2 W} \\
& =-\frac{L}{a} \sum_{m=1}^{\infty} \frac{(-1)^{m+1}}{m(2 m+1)}\left(\frac{W}{v_{F}}\right)^{2 m} \\
& =-L / l_{l o c},
\end{aligned}
$$

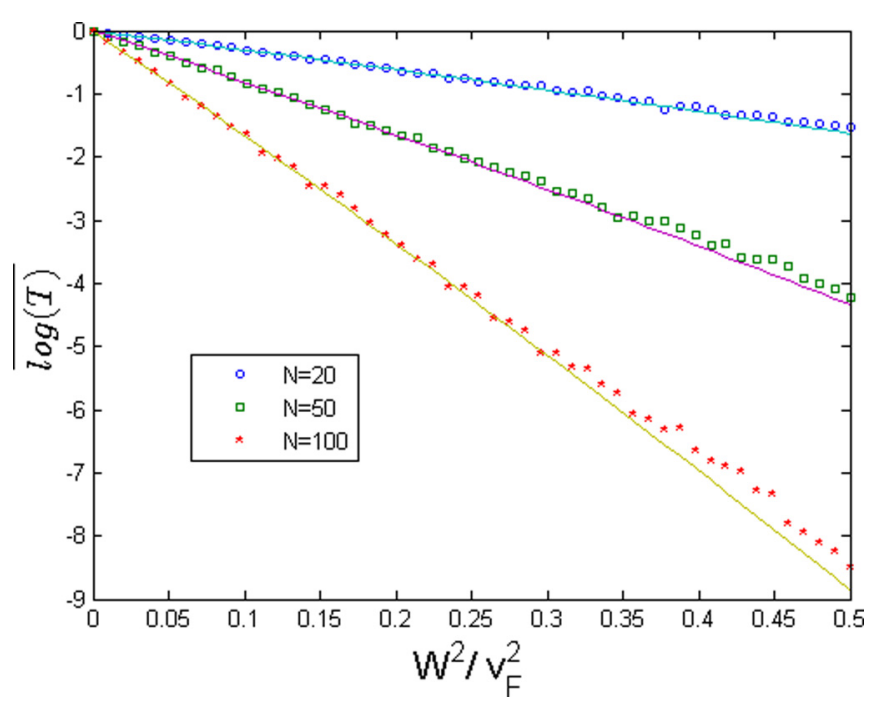

FIG. 6. (Color online) $\overline{\log (T)}$ for different system sizes, $N=20$, 50,100 with $k a=0.25 \pi$ using uniform distribution $[-W, W]$ is numerically computed (dots) and compared with the analytic results (lines), Eq. (49). Despite ignoring multiple scatterings from different impurities, as a leading order approximation the analytic result works well beyond its perturbative regime $W^{2} / v_{F}^{2}<1 / N$. The disorder averaging with 1000 realizations is performed.

where $L=(N-2) a$ is the length of disordered regime in the wire. The next dominant correction with nonzero argument of the cosine function is discussed in the Appendix. Such rotating terms could be important for the wavelength commensurate with lattice spacing; otherwise the sum of rotating terms will average out to zero in general. The localization length of the system is found as

$l_{l o c} / a=\left[\log \left(1+\frac{W^{2}}{v_{F}^{2}}\right)-\frac{v_{F}}{i W} \log \left(\frac{1+i W / v_{F}}{1-i W / v_{F}}\right)-2\right]^{-1}$,

which is plotted in Fig. 6 compared with computational results that take into account all nonzero arguments of the cosine function, showing reasonably good matching for different system sizes. The very first term of the inverse localization length $a / l_{l o c} \simeq W^{2} / 3 v_{F}^{2}$ and this is inconsistent with previous findings using perturbation approaches $[42,43]$.

It is worthwhile to compute the variance of the inverse localization length, in order to check whether $(\log T) / L$ is really statistically well behaved. Numerically [39] and analytically [42], it was found that the variance is proportional to the inverse square root of system size. The first leading contribution for the variance comes from the $m=1$ term, Eq. (47), and the computation is directly following:

$$
\begin{aligned}
\overline{\left(\frac{\log T}{L}\right)^{2}} & =\overline{\left(\frac{2}{L} \sum_{i=2}^{N-1} \frac{\alpha_{i}}{v_{F}}\right)^{2}}+O\left(\frac{W^{4}}{L}\right) \\
& =\frac{4}{L} \frac{a}{l_{l o c}}+O\left(\frac{W^{4}}{L}\right),
\end{aligned}
$$




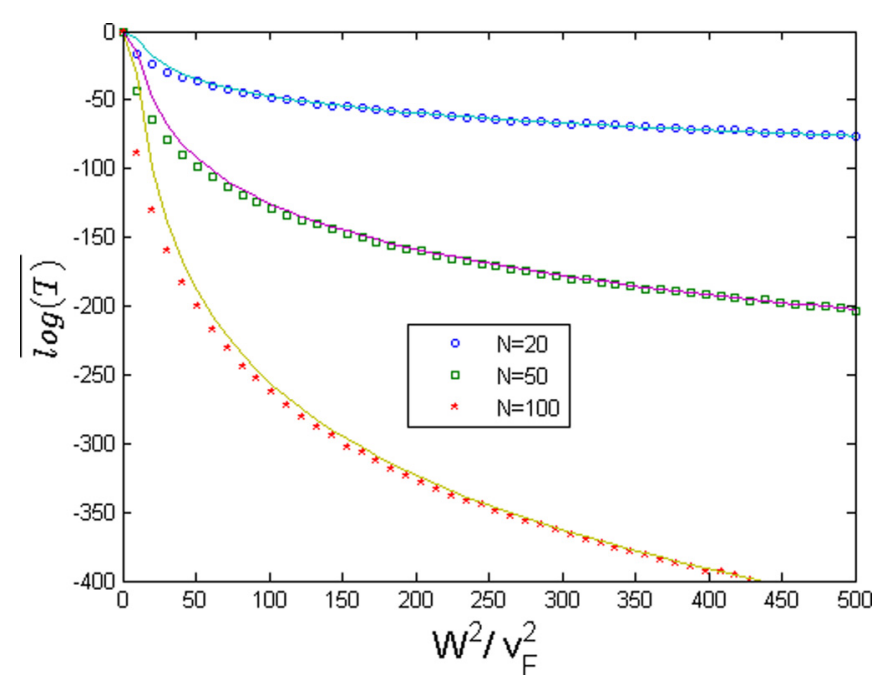

FIG. 7. (Color online) $\overline{\log (T)}$ in strong disorder strength limit with $k a=0.25 \pi$ is numerically computed (dots) and compared with analytic approximation (lines) according to Eq. (56). They match well in the strong-disorder regime, while in the weak-disorder regime a deviation is present since the perturbation parameter is now $\lambda=v_{F}^{2} / W^{2}$, and the range of impurity strength is $[-W, W]$ instead of $[-1 / W, 1 / W]$. The disorder averaging with 1000 realizations is performed.

where $a / l_{l o c}=W^{2} / 3 v_{F}^{2}$ up to the order of $W^{2}$. Therefore, we obtain the previously known relation [39,42]:

$$
\frac{\operatorname{var}\left(l_{l o c}^{-1}\right)}{l_{l o c}^{-1}}=\frac{4}{L / a}+O\left(\frac{W^{4}}{L}\right),
$$

confirming that the $\log T / L$ a variable following the central limit theorem.

Let us end this section with the disorder averaging of opposite limit. For "strong" disorder strength, we take $v_{F} / W$ as a perturbation expansion parameter. We are careful to use the term "strong," since our expression based on the picture of two chiral modes would not be valid as the energy scale brought by impurities is comparable or larger than energy band width of a clean wire. For more discussion of the "strong" disorder limit, we refer to Sec. III A. Having the elements of matrix $1 \alpha G$ large, the transmission is

$$
\begin{aligned}
T & =\left|\frac{1}{\operatorname{det}[I+\alpha G]}\right|^{2} \simeq\left|\frac{1}{\operatorname{det}[\alpha G]}\right|^{2}, \\
\log (T) & =C+\sum_{i=2}^{N-1} \log \left(\frac{v_{F}^{2}}{\alpha_{i}^{2}}\right),
\end{aligned}
$$

where $C=-2 \log \left|\operatorname{det}\left[v_{F} G\right]\right|$. Therefore, the average of the $\log T$ in the uniform distribution of disorder is straightforward:

$$
\overline{\log (T)} \simeq C-2(N-2)\left[\log \left(\frac{W}{v_{F}}\right)-1\right],
$$

which is plotted in Fig. 7 for different system size and shows a good agreement. This is also inconsistent with a previous study by Stone et al . [42] in the strong-disorder limit. It is also interesting to compare this strong-disorder limit expression to the weak-disorder one that the second term of Eq. (56) is obtained from Eq. (50) in the $W / v_{F} \gg 1$ limit.

\section{Resistance: $\bar{R}=\overline{1 / T}$}

There have been many studies of the resistance of disordered 1d wires [32-36]. Like the transmission, which was discussed earlier, the resistance is not a statistically well behaved quantity since its fluctuations increase faster than its average with system size. Nevertheless, various approaches have been used to compute the moments of the resistance $[34,35,40,44]$, and the scaling behavior of resistance with system size attracted a great deal of attention [33,35,39]. Our formalism does not offer significant advantages for computing the disorder average of the resistance; nonetheless, we carry out the calculation for the sake of completeness of the formulation developed in this paper.

The resistance of $1 \mathrm{~d}$ wire is proportional to the inverse transmission $1 / T$. Thus, let us focus on the disorder averaging of $1 / T$ for simplicity. Directly from Eq. (35) the inverse transmission can be expressed by the determinant of the matrices:

$$
\begin{aligned}
1 / T & =\frac{1}{\mathcal{G}_{N 1} \mathcal{G}_{N 1}^{*}} \\
& =\frac{\operatorname{det}\left[G^{-1} G^{*-1}-\alpha G^{*-1}-G^{-1} \alpha+\alpha \alpha\right]}{\operatorname{det}\left[G^{-1} G^{*-1}\right]},
\end{aligned}
$$

with $G^{-1}=1 /\left(G_{R}+G_{L}\right)$, which is the Hamiltonian of clean $1 \mathrm{~d}$ wire and therefore a tridiagonal matrix. Hence, the matrix inside the determinant in the numerator in Eq. (58) is essentially an extended version of a tridiagonal matrix which has five nonzero elements along the diagonal element instead of three as in Eq. (35), with the elements

$$
\begin{aligned}
D_{n, n} & =\left(\alpha_{n}-E\right)^{2}+2 t^{2}, \\
D_{n+1, n} & =D_{n, n+1}=t\left(\alpha_{n}+\alpha_{n+1}\right)-2 t E, \\
D_{n+2, n} & =D_{n, n+2}=t^{2} .
\end{aligned}
$$

Here $D_{1,1}=D_{N, N}=2 t^{2}, \alpha_{1}=\alpha_{N}=t e^{-i k a}$, and we place impurities at $i=6,10,14, \ldots, N-5$ so that transfer matrices are not impurity correlated. As a result, the recursion relation involving a transfer matrix can be written using six elements:

$$
\left(\begin{array}{l}
d_{4(m+1)+1} \\
d_{4(m+1)+2} \\
d_{4(m+1)+3} \\
d_{4(m+1)+4} \\
X_{4(m+1)+2} \\
X_{4(m+1)+3}
\end{array}\right)=T_{6 \times 6}^{m}\left(\begin{array}{l}
d_{4 m+1} \\
d_{4 m+2} \\
d_{4 m+3} \\
d_{4 m+4} \\
X_{4 m+2} \\
X_{4 m+3}
\end{array}\right),
$$

where $d_{4 m+j}$ is the determinant of the upper left square matrix of $D$ up to the $(4 m+1)$ th row and column. $X_{n}$ are also the determinants of a similar matrix which is required to construct the transfer matrix (see Fig. 8):

$$
\begin{aligned}
X_{n}= & D_{n, n+1} d_{n-1}-D_{n-1, n+1} D_{n, n-1} d_{n-2} \\
& -D_{n-1, n+1} D_{n-2, n} X_{n-2} .
\end{aligned}
$$

From this recursion relation one can build the train of transfer matrices and perform disorder averaging for each transfer matrix separately. 
(a)

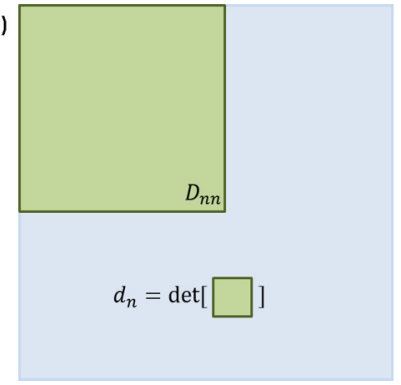

(b)

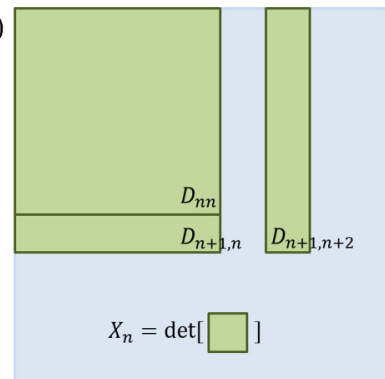

FIG. 8. (Color online) Describing the elements of the determinant in Eq. (62). (a) $d_{n}$ is the determinant of Green's square block up to element $D_{n n}$. (b) $X_{n}$ is the determinant of the Green's blocks collection.

To have an analytic description, we look for the exponents of the $1 / T$, which are related to the eigenvalues of the transfer matrix. The characteristic equation of the disorder-averaged transfer matrix $T_{6 \times 6}^{m}$ has one trivial eigenvalue, unity, and the others are from a quintic equation whose general solution is not analytically available. Here we show the computation of "analytic" and numeric results in Fig. 9. The plot shows the exponential increase of the resistance of disordered wire with the system size.

\section{EXAMPLE: QUANTUM HALL FLUID}

Another natural application for the det formula is the quantum Hall system. The application of the det formula is rather straightforward as it contains an explicit chiral model along the boundary. Consider the system described in Fig. 10 where impurities near the boundary may scatter the chiral mode, and impurities are also connected with each other in a random fashion. In the single-particle picture, the conductance of the chiral mode is expected to be quantized; however, the

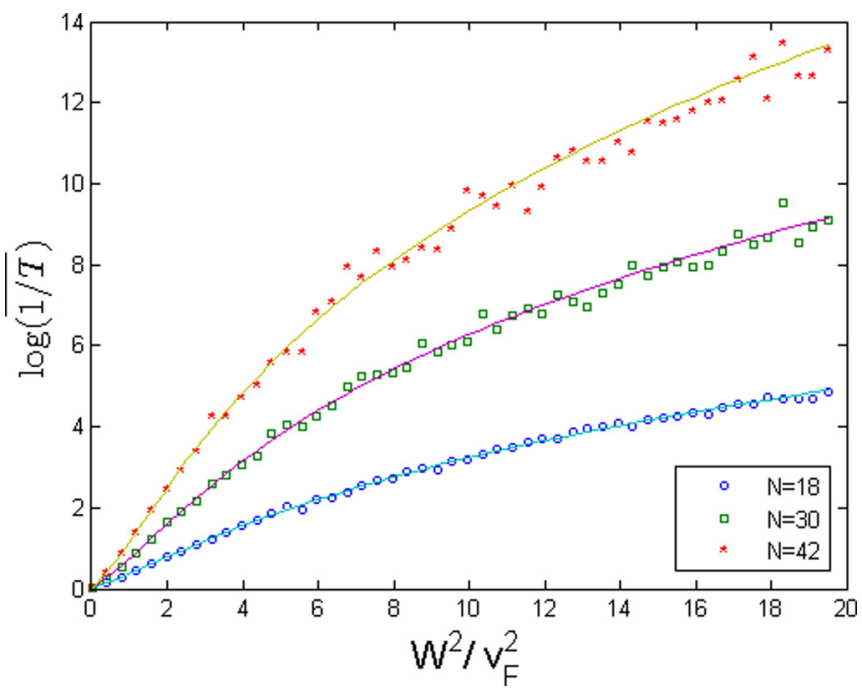

FIG. 9. (Color online) $\log \overline{1 / T}$ for different system sizes $N=$ 18,30 , and 42 with $k a=0.45 \pi$ is computed numerically (dots) by disorder averaging of 2000 realization. And, the analytic disorder averaging (lines) is performed exactly using transfer matrix, though its eigenvalues cannot be expressed in a closed form.

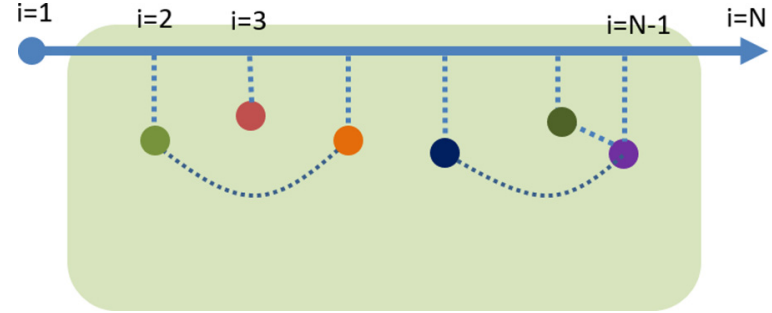

FIG. 10. (Color online) A chiral mode in quantum Hall system with an insulating bulk. Multiple impurities with random couplings near the edge are encountered by the chiral mode, and the accumulated phase is estimated with the det formula.

Green's function will possess a system-specific phase factor due to the scattering from the impurities. These nontrivial phase factors in quantum Hall systems can be probed by different types of interferometers [45-49]. Our purpose in this section is to estimate the accumulated phase throughout the system.

Let us introduce the Green's function, $G_{B}$, of quantum dots with random coupling, the coupling matrix $T$ between chiral mode and quantum dots, and $\mathcal{T}=\hat{U}_{s t} V / 2 v_{F}$, as done in Sec. II. The direct application of the det formula gives

$$
\begin{aligned}
\mathcal{G}_{N 1} & =\frac{e^{i(N-1) k a}}{i v_{F}} \frac{\operatorname{det}\left[I-G_{L 0}^{-1} \mathcal{T} G_{B} \mathcal{T}^{\dagger}\right]}{\operatorname{det}\left[I+\mathcal{T} G_{B} \mathcal{T}^{\dagger} G_{R 0}^{-1}\right]} \\
& =\frac{e^{i(N-1) k a}}{i v_{F}} \frac{e^{\operatorname{Tr} \log \left[I-G_{L 0}^{-1} \mathcal{T} G_{B} \mathcal{T}^{\dagger}\right]}}{e^{\operatorname{Tr} \log \left[I-\left(G_{L 0}^{-1} \mathcal{T} G_{B}^{\dagger} \mathcal{T}^{\dagger}\right)^{\dagger}\right]}},
\end{aligned}
$$

where in the first equality the order of matrix multiplication is changed within the determinant of denominator, then in the second equality $\left(G_{R 0}^{-1}\right)^{\dagger}=-G_{L 0}^{-1}$ is used. Having a symmetric form, diagonalization helps to further simplify:

$$
G_{L 0}^{-1} \mathcal{T} G_{B} \mathcal{T}^{\dagger}=U^{-1} \Lambda U
$$

The bulk Green's function $G_{B}$ may, in general, not be a Hermitian matrix in an open, lossy, system. However, when the bulk states are insulating and do not contain propagating states to the exterior, the bulk Green's function becomes real and symmetric, $G_{B}^{\dagger}=G_{B}$. As a result, the renormalized Green's function is now reduced to

$$
\mathcal{G}_{N 1}=\frac{e^{i(N-1) k a}}{i v_{F}} \exp \left(\sum_{n} \log \left[\frac{1-\lambda_{n}}{1-\lambda_{n}^{*}}\right]\right),
$$

which gives a succinct expression for the accumulated phase due to random impurity scatterings in terms of the eigenvalues of the matrix Eq. (65). We expect this expression to be useful in similar systems.

\section{CONCLUSION}

In this paper we introduced a closed form expression for the propagator of a chiral mode coupled to a bulk. For a given bulk Green's function and coupling matrix to the chiral mode, we were able to express the renormalized chiral Green's function by the ratio of determinants. No assumption is made on the bulk Green's function; therefore, the formula is applicable to a system with and without nonlocalized bulk modes. 
Using a disordered 1d quantum wire as an example, we demonstrated how nonchiral $1 \mathrm{~d}$ systems can be modeled in terms of chiral modes, and computed the average transmission coefficient, its inverse, and logarithm. The det formula was shown to be especially powerful in performing disorder averaging for different transport quantities. A similar trick is expected to work for quasi-1d and topological metal systems.

As a second example, the phase accumulated by the chiral mode is conveniently estimated in a quantum Hall system with random impurities. This formula may be used to describe the interference pattern in quantum Hall interferometers as a function of chemical potential of the bulk and other system characteristics.

\section{ACKNOWLEDGMENTS}

It is a pleasure to acknowledge useful discussions with Matthew Hastings and Konstantin Efetov as well as funding from the IQIM, an NSF center, supported by the Moore Foundation, and from DARPA through FENA. I.K. acknowledges financial support from NSF CAREER Award No. DMR-0956053.

\section{APPENDIX}

Localization length was computed by averaging the logarithm of transmission coefficient for different disorder realizations. We computed the main contribution in Eq. (49) after ignoring the rotating terms. Up to the second order in disorder strength, the analytics and numerical results are in good agreement (Fig. 6) in the weak-disorder regime. However, as soon as the fourth and higher orders become important at moderate disorder strength, the contribution from the rotating term should be taken into account. In this appendix we compute the leading correction of the rotating term by allowing the indices $n_{i}$ to be different.

\section{Localization length: Next order}

In Sec. III C 2, we obtained the leading order of disorderaveraged $\log (T)$, assuming all indices in Eq. (47) are the same: $n_{1}=n_{2}=\cdots=n_{m}$. For the next leading correction, we allow the indices to be two different indices: $n_{1}, \ldots, n_{m} \in n_{i}, n_{j}$ with $i \neq j$. Then all possible terms of order m can be expressed by the help of a $2 \times 2$ transfer matrix form for even $m$ :

$$
\begin{aligned}
& \sum_{n_{1}, \cdots, n_{m}}\left(\prod_{i=1}^{m} \frac{\alpha_{n_{i}}}{v_{F}}\right) \operatorname{Re}\left[e^{i k a \sum_{i=1}^{m}\left|n_{i+1}-n_{i}\right|}\right] \\
& =\sum_{n_{1}, n_{2}} \operatorname{Re} \operatorname{Tr}\left[\left(\begin{array}{cc}
\alpha_{n_{1}} & \alpha_{n_{1}} e^{i k \Delta} \\
\alpha_{n_{2}} e^{-i k \Delta} & \alpha_{n_{2}}
\end{array}\right)^{m}-\left(\begin{array}{cc}
\alpha_{n_{1}}^{m} & 0 \\
0 & \alpha_{n_{2}}^{m}
\end{array}\right)\right],
\end{aligned}
$$

where $\Delta=\left|n_{1}-n_{2}\right| a$ is the distance between two impurity sites. Now the problem is reduced to finding the eigenvalue of the transfer matrix:

$$
\lambda_{ \pm}=\frac{\alpha_{n_{1}}+\alpha_{n_{2}}}{2} \pm \sqrt{\frac{\left(\alpha_{n_{1}}-\alpha_{n_{2}}\right)^{2}}{4}+\alpha_{n_{1}} \alpha_{n_{2}} e^{2 i k \Delta}}
$$

By using the prefactor in Eq. (47) and inserting the eigenvalues:

$$
\begin{aligned}
\log \left(T_{(m)}\right) & =\sum_{n_{1}, n_{2}} \frac{(-1)^{m}}{m v_{F}^{m}} 2 \operatorname{Re}\left[\lambda_{+}^{m}+\lambda_{-}^{m}-\alpha_{n_{1}}^{m}-\alpha_{n_{2}}^{m}\right] \\
& =2 \operatorname{Re} \sum_{n_{1}, n_{2}} \log \left[\frac{\left(1+\frac{\lambda_{+}}{v_{F}}\right)\left(1+\frac{\lambda_{-}}{v_{F}}\right)}{\left(1+\frac{\alpha_{n_{1}}}{v_{F}}\right)\left(1+\frac{\alpha_{n_{2}}}{v_{F}}\right)}\right] \\
& =2 \operatorname{Re} \sum_{n_{1}, n_{2}} \log \left[1-\frac{\alpha_{n_{1}} \alpha_{n_{2}} e^{2 i k \Delta} / v_{F}^{2}}{\left(1+\frac{\alpha_{n_{1}}}{v_{F}}\right)\left(1+\frac{\alpha_{n_{2}}}{v_{F}}\right)}\right],
\end{aligned}
$$

which is the generating function for the correction of two indices to all orders for a particular disorder realization. For example, the first correction appears in the fourth order with nonzero argument of cosine function. By expanding the logarithm in series of $\alpha_{i} / v_{F}$, then disorder averaging:

$$
\begin{aligned}
\overline{\log \left(T_{(4)}\right)} & =\operatorname{Re} \sum_{n_{1}, n_{2}} \frac{\overline{\alpha_{n_{1}}^{2} \alpha_{n_{2}}^{2}}\left(4 e^{2 i k \Delta}+2 e^{4 i k \Delta}\right)}{-2 v_{F}^{2}} \\
& =\frac{\left\langle\alpha^{2}\right\rangle^{2}}{-v_{F}^{4}} \sum_{\Delta / a=1}^{L / a-1} \frac{L-\Delta}{a}[2 \cos (2 k \Delta)+\cos (4 k \Delta)] \\
& =\frac{\left\langle\alpha^{2}\right\rangle^{2}}{v_{F}^{4}}\left[\frac{3 L}{2 a}-\frac{\sin ^{2}(k L)}{\sin ^{2}(k a)}-\frac{\sin ^{2}(2 k L)}{2 \sin ^{2}(2 k a)}\right],
\end{aligned}
$$

where $L=(N-2) a$ is the length of disordered regime. One can see that the term in the square bracket may not be small depending on the wave number $k$. But, the term goes to zero upon the integration over $k$. This is the case for all higher order corrections. By carefully performing the expansion of

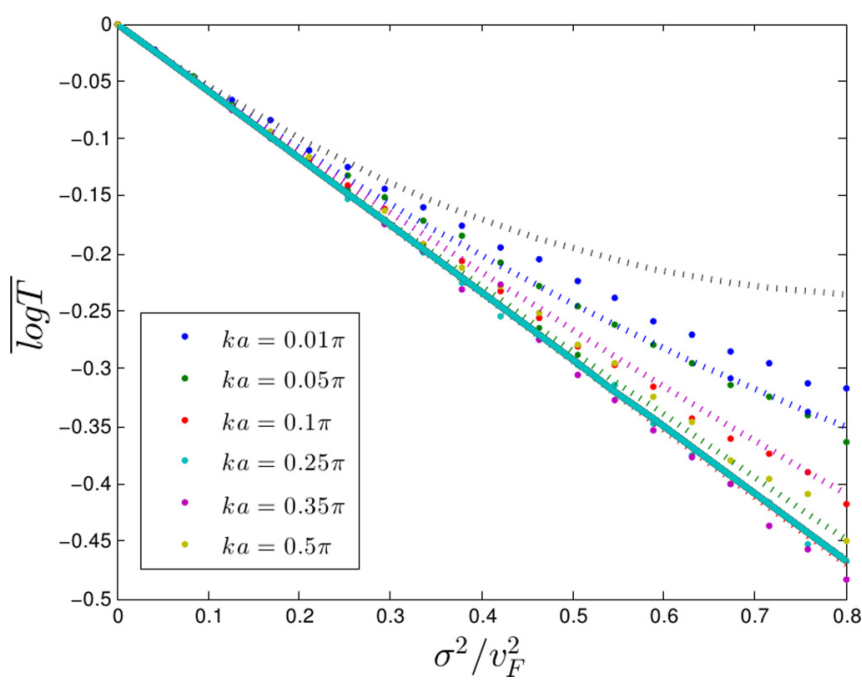

FIG. 11. (Color online) Disorder-averaged $\log T$ is plotted using the exact expression (dots) from Eq. (35), the single index contribution (solid line) from Eq. (49), and the result which includes the doubleindex correction (dotted line), Eq. (A3). At moderate disorder the single-index contribution fails to reproduce the exact results for the various wave numbers. The double-index correction, however, does manage to account for wave-number dependence except for the smallest wave number. 
the generating function, the general expression for $m$ th-order correction of two indices can be deduced:

$$
\begin{gathered}
\overline{\log \left(T_{(m)}\right)}=\frac{1}{m} \sum_{n=2, \text { even }}^{m-2} \sum_{l=1}^{l_{u p}} \frac{1}{n+1} \frac{(-1)^{m / 2+1}}{m-n+1}\left(\frac{W}{v_{F}}\right)^{m} \\
\times\left(\begin{array}{c}
m-n \\
l
\end{array}\right)\left(\begin{array}{l}
n \\
l
\end{array}\right) l\left(\frac{1}{n}+\frac{1}{m-n}\right)\left[\frac{L}{a}-\frac{\sin ^{2}(k L l)}{\sin ^{2}(k a l)}\right],
\end{gathered}
$$

where $l_{u p}=\min (n, m-n)$ and indices $m$ and $n$ are even integers. Figure 11 shows the contribution of $\overline{\log \left(T_{(m)}\right)}$ in addition to the leading order with single index, Eq. (49) (solid line). The dots are from the numerical result averaged over 4000 disorder realizations. The difference between dotted lines and the solid line are corrections made by double index terms for different wave numbers. $\overline{\log T}$ is independent of wave number in the weak-disorder limit and they converge to a single line, Eq. (49).
[1] C. L. Kane and E. J. Mele, Phys. Rev. Lett. 95, 146802 (2005).

[2] L. Fu and C. L. Kane, Phys. Rev. B 74, 195312 (2006).

[3] J. E. Moore and L. Balents, Phys. Rev. B 75, 121306 (2007).

[4] T. Fukui, T. Fujiwara, and Y. Hatsugai, J. Phys. Soc. Jpn. 77, 123705 (2008).

[5] X.-L. Qi, T. L. Hughes, and S.-C. Zhang, Phys. Rev. B 78, 195424 (2008).

[6] R. Roy, Phys. Rev. B 79, 195321 (2009).

[7] Z. Wang, X.-L. Qi, and S.-C. Zhang, New J. Phys. 12, 065007 (2010).

[8] X. G. Wen, Phys. Rev. B 41, 12838 (1990).

[9] A. Stern, Ann. Phys. 323, 204 (2008).

[10] D. C. Tsui, H. L. Stormer, and A. C. Gossard, Phys. Rev. Lett. 48, 1559 (1982).

[11] K. v. Klitzing, G. Dorda, and M. Pepper, Phys. Rev. Lett. 45, 494 (1980).

[12] C. Xu and J. E. Moore, Phys. Rev. B 73, 045322 (2006).

[13] C. Wu, B. A. Bernevig, and S.-C. Zhang, Phys. Rev. Lett. 96, 106401 (2006).

[14] V. Cheianov and L. I. Glazman, Phys. Rev. Lett. 110, 206803 (2013).

[15] J. Li, R.-L. Chu, J. K. Jain, and S.-Q. Shen, Phys. Rev. Lett. 102, 136806 (2009).

[16] C. W. Groth, M. Wimmer, A. R. Akhmerov, J. Tworzydło, and C. W. J. Beenakker, Phys. Rev. Lett. 103, 196805 (2009).

[17] H.-M. Guo, G. Rosenberg, G. Refael, and M. Franz, Phys. Rev. Lett. 105, 216601 (2010).

[18] D. L. Bergman and G. Refael, Phys. Rev. B 82, 195417 (2010).

[19] A. Junck, K. W. Kim, D. L. Bergman, T. Pereg-Barnea, and G. Refael, Phys. Rev. B 87, 235114 (2013).

[20] T. Brandes and S. Kettemann, Anderson Localization and Its Ramifications: Disorder, Phase Coherence, and Electron Correlations, Lecture Notes in Physics Vol. 630 (Springer, Berlin, 2003).

[21] E. Abrahams, P. W. Anderson, D. C. Licciardello, and T. V. Ramakrishnan, Phys. Rev. Lett. 42, 673 (1979).

[22] F. Delyon, H. Kunz, and B. Souillard, J. Phys. A: Math. Gen. 16, 25 (1983).
[23] K. Efetov, Supersymmetry in Disorder and Chaos (Cambridge University Press, Cambridge, 1999).

[24] F. Wegner, Z. Phys. B: Condens. Matter 35, 207 (1979).

[25] F. Iglói and C. Monthus, Phys. Rep. 412, 277 (2005).

[26] P. W. Anderson, Phys. Rev. 109, 1492 (1958).

[27] A. D. Stone and A. Szafer, IBM J. Res. Dev. 32, 384 (1988).

[28] D. Thouless, J. Phys. C 5, 77 (1972).

[29] T. Fukuhara, P. Schauß, M. Endres, S. Hild, M. Cheneau, I. Bloch, and C. Gross, Nature (London) 502, 76 (2013).

[30] P. A. Lee, A. D. Stone, and H. Fukuyama, Phys. Rev. B 35, 1039 (1987).

[31] C. W. J. Beenakker, Rev. Mod. Phys. 69, 731 (1997).

[32] E. Abrahams and M. J. Stephen, J. Phys. C 13, L377 (1980).

[33] P. W. Anderson, D. J. Thouless, E. Abrahams, and D. S. Fisher, Phys. Rev. B 22, 3519 (1980).

[34] N. Kumar, Phys. Rev. B 31, 5513 (1985).

[35] P. D. Kirkman and J. B. Pendry, J. Phys. C 17, 4327 (1984).

[36] A. D. Stone, J. D. Joannopoulos, and D. J. Chadi, Phys. Rev. B 24, 5583 (1981).

[37] J. B. Pendry, Adv. Phys. 43, 461 (1994).

[38] P. Erdös and R. Herndon, Adv. Phys. 31, 65 (1982).

[39] B. S. Andereck and E. Abrahams, J. Phys. C 13, L383 (1980).

[40] P. A. Mello, J. Math. Phys. 27, 2876 (1986).

[41] J. Sak and B. Kramer, Phys. Rev. B 24, 1761 (1981).

[42] A. D. Stone, D. C. Allan, and J. D. Joannopoulos, Phys. Rev. B 27, 836 (1983).

[43] B. Kramer and A. MacKinnon, Rep. Prog. Phys. 56, 1469 (1993).

[44] A. Abrikosov, Solid State Commun. 37, 997 (1981).

[45] C. de C. Chamon, D. E. Freed, S. A. Kivelson, S. L. Sondhi, and X. G. Wen, Phys. Rev. B 55, 2331 (1997).

[46] D. E. Feldman and A. Kitaev, Phys. Rev. Lett. 97, 186803 (2006).

[47] D. E. Feldman, Y. Gefen, A. Kitaev, K. T. Law, and A. Stern, Phys. Rev. B 76, 085333 (2007).

[48] A. R. Akhmerov, J. Nilsson, and C. W. J. Beenakker, Phys. Rev. Lett. 102, 216404 (2009).

[49] W. Bishara and C. Nayak, Phys. Rev. B 77, 165302 (2008). 